\title{
Analysis of the End Regions of the CERN Lead-Ion 4-rod RFQ
}

\author{
V.A. Andreev \\ ITEP, Bolshaja Cherjomushkinskaja 25, 117259 Moscow, Russia \\ A. Lombardi, G. Parisi \\ INFN, Laboratori Nazionali di Legnaro, via Romea 4, I-35020 Legnaro (PD), Italy \\ M. Vretenar \\ CERN, PS Division, CH-1211 Geneva 23, Switzerland
}

\section{Abstract}

A 4-rod-like Radio Frequency Quadrupole for the new CERN lead-ion injector will be built at the Laboratori Nazionali di Legnaro, Italy [1].

By means of 3D simulations with MAFIA codes [2] and measurements on a cold model, an asymmetry was found in the gap between the end plate and electrodes, yielding a non-zero longitudinal component $E_{z}$ on the axis of the RFQ and therefore a potential barrier in the gap.

In this paper an explanation is given for this fact and possible solutions are proposed for end cell tuning, which allow to eliminate the undesirable $E_{z}$ component and also to obtain good inter-electrode voltage distribution along the structure. Results of MAFIA calculations and cold model measurements are shown.

\section{INTRODUCTION}

We considered the structure shown in Fig. 1, representing a part (4 supports instead of 14) of the Lead-Ion RFQ as modelled by MAFIA codes; the end plates are not shown for the sake of clearness. It is a 4-rod structure, similar to the ones developed in Frankfurt [3] but having double symmetric supports and "vane-like" shaped rods.

By means of the MAFIA codes, it was found that the voltages at the end of the electrodes are not balanced with respect to the external tank; actually, at their end, one pair is near the full potential $V_{e}$, oscillating at $\mathrm{RF}$, while the other pair remains near the ground potential.

This doesin't affect the quadrupolar symmetry inside the accelerating channel, but determines on the axis a potential difference $V_{e} / 2$ between the end of the electrodes and the end plates. As a consequence, a longitudinal component $E_{z}$ of the electric field arises, and sach region behaves like a RF gap that may accelerate the particles passing throughout it.

\section{ANALYSIS OF THE PHENOMENON}

We evaluated the $\vec{B}$ field distribution for the above structure. In Fig. 2 the longitudinal section at the vertical symmetry plane is shown; Fig. 3 displays two transverse sections: through a support and in the center of a cell.

We can see that middle cells are filled with magnetic field, whose lines are closing around the supports and the electrodes, while there is almost no field in the end cells. This absence, confirmed by measurements on cold models, is not peculiar of our "double support" structure, although



Fig. 1: MAFIA plot of the CERN Lead-Ion 4-rod RFQ

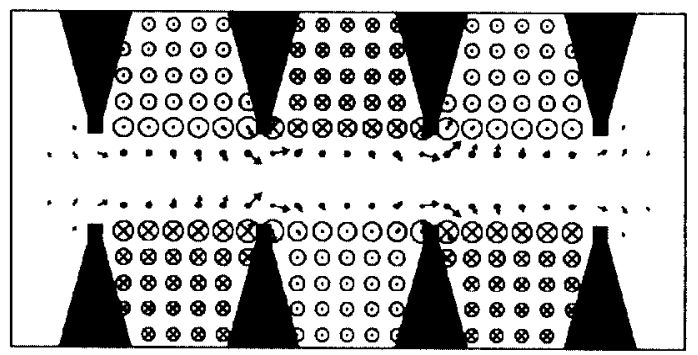

Fig. 2: MAFIA plot of $\vec{B}$ field (longitudinal section)



Fig. 3: MAFIA plots of $\vec{B}$ field (transverse sections)

it depends on the geometry used and in our case happens to be more evident than in other RFQ's.

In order to understand this phenomenon, we investigate the behaviour of our RFQ by considering the electrical properties of a chain of five coupled oscillators: from the RF point of view, it consists of coupled resonant cells, all equal to each other except the ending ones, that have half length and are terminated with a metallic plate, separated by a gap from the end of the electrodes.

When the middle cell is excited (by a feeder loop) it resonates at a certain $\omega_{0}=1 / \sqrt{L_{0} C_{0}}$, where $L_{0}$ and $C_{0}$ are its equivalent inductance and capacitance. The adjacent cells have the same $L_{0}$ and $C_{0}$ and are strongly coupled, while 


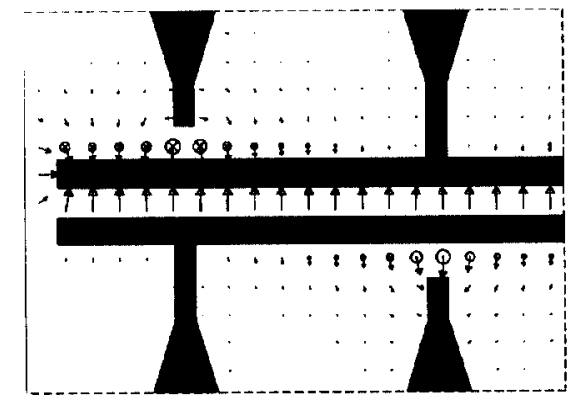

Fig. 4: MAFIA plot of $\vec{E}$ field (detail)

the end cells have half the inductance $\left(L_{\text {end }}\right)$ and a lower capacitance $\left(C_{\text {end }}\right.$ is half of $C_{0}$ plus the small capacitance between the end plate and electrodes). Their resonant frequency is therefore much higher and the consequence is that there is no $\vec{B}$ field (or just a small one) and no induced currents (or small ones) in the ending regions, due to the absence of a time-varying magnetic flux.

Another complementary effect is that the interelectrode voltage $V_{e}$ drops in the middle of the structure (see Fig. 9-left), also due to the difference in the end cells behaviour.

In Fig. 4 it is shown a detail of $\vec{E}$ field in a longitudinal section through a pair of electrodes; we can see that the potential symmetry condition $\left(+V_{e} / 2\right.$ on one pair of electrodes and $-V_{e} / 2$ on the other one, relatively to the end plate) is not fulfilled at the end of the electrodes.

This is also illustrated by Fig. 7-left, where a detail of $\vec{E}$ field in the gap is shown (in a transverse section near end plate): on the axis, MAFIA codes evaluated a longitudinal component $E_{z}$ which integrated along the gap leads to a voltage $V_{e} / 2$.

To explain this phenomenon, let us consider the semplified equivalent lumped circuit of an end cell shown in Fig. 5-left, where four rods are presented, for simplicity, as a two-wire line. As seen before, there are almost nor $\vec{B}$ field nor currents in the ending regions, so that we can evaluate potentials $V_{\mathrm{A} 0}$ and $V_{\mathrm{B} 0}$ of the points $\mathrm{A}$ and $\mathrm{B}$ with respect to point 0 (ground) as:

$$
\begin{gathered}
V_{\mathrm{A} 0} \approx V_{\mathrm{AC}^{\prime}}+V_{\mathrm{C}^{\prime} \mathrm{C}}+V_{\mathrm{C} 0} \approx 0 \\
V_{\mathrm{B} 0} \approx V_{\mathrm{BC}}+V_{e}+V_{\mathrm{C}^{\prime} \mathrm{C}}+V_{\mathrm{C} 0} \approx V_{e}
\end{gathered}
$$

While beam dynamics calculations were carried on in order to analyse the effect of this asymmetry on the heavy ion beam [4], we concentrated on finding an end cell configuration achieving the condition:

$$
V_{\mathrm{A} 0}=-\frac{1}{2} V_{e} \quad V_{\mathrm{B} 0}=+\frac{1}{2} V_{e}
$$

To reach such goal on our RFQ, different versions of end cell tuning were simulated using MAFIA codes and the best one was then tested on a cold model.

\section{POSSIBLE SOLUTIONS}

In order to cope with the above effects, which are due to the difference between middle and end cells, we need to tune the latter, so to have there a proper electromagnetic field. Thus, the $E_{z}$ component arising in the gap between end plate and electrodes disappears.

Our approach follows from the one proposed by Pirkl [5], attempting to include the equivalent terminating reactance elements at the end of the electrodes relatively to end plate. We analyse his proposal from the point of view of end cells tuning, i.e. of clectromagnetic fields in the end cells.
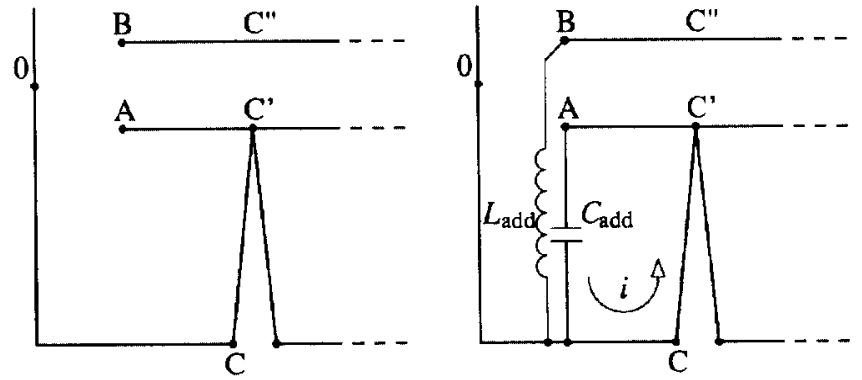

Fig. 5: Equivalent lumped circuits

As a first attempt, we can try to connect "hot" ends of electrodes (B) to the external tank by means of conductive strips, i.e. we add an inductance $L_{\text {add }}$ (Fig. 5-right). The choice of $L_{\text {add }}$ is limited by two conditions:

- To reach a balance between the cells, the resonant frequency of the end cell should be equal to the middle cell one:

$$
\left(L_{\text {end }}+L_{\text {add }}\right) C_{\text {end }}=\omega_{0}^{-2}
$$

As in our case the end cell length is half of the middle one, for symmetry reasons we have $L_{\text {end }}=\frac{1}{2} L_{0}$ and $C_{\text {end }}=\frac{1}{2} C_{0} ;$ thus, to fulfill (4), it must be:

$$
L_{\text {add }}=\frac{3}{2} L_{0}
$$

- In order to fulfill (3), the voltage drop on $L_{\text {add }}$ should be equal (in module) to the one on $L_{\text {end }}$. As the same current passes through both inductances, it means:

$$
L_{\text {add }}=L_{\text {end }}=\frac{1}{2} L_{0}
$$

It is not possible to satisfy both conditions (3) and (4) changing only the inductance of the last cell, in fact we obtained (5) and (6) which are incompatible. Therefore one has to act simultaneously on both inductance and capacitance, adding $L_{\text {add }}$ at the "hot" electrodes and $C_{\text {add }}$ at the "cold" ones. Inductance can be further increased by changing the shape of the last support; capacitance by a vane-connecting ring or adding some plates between the end of the "cold" electrodes (A) and the end plate, i.e. a capacitance $C_{\text {add }}$.

By MAFIA simulations, many different combinations of strips, plates and rings were tested on the cold model geometry. Modifying their dimension and position, each version allowed to obtain a good longitudinal $V_{e}$ distribution (flatness within $\pm 1 \%$ ) and to reduce $4 \div 15$ times the 


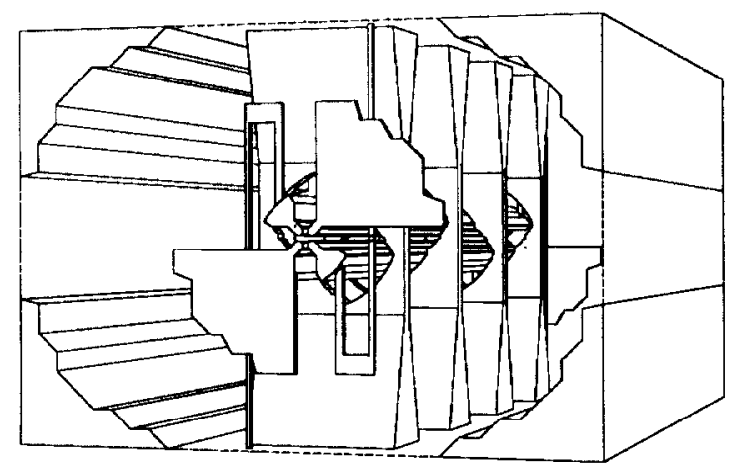

Fig. 6: MAFIA plot of a possible solution
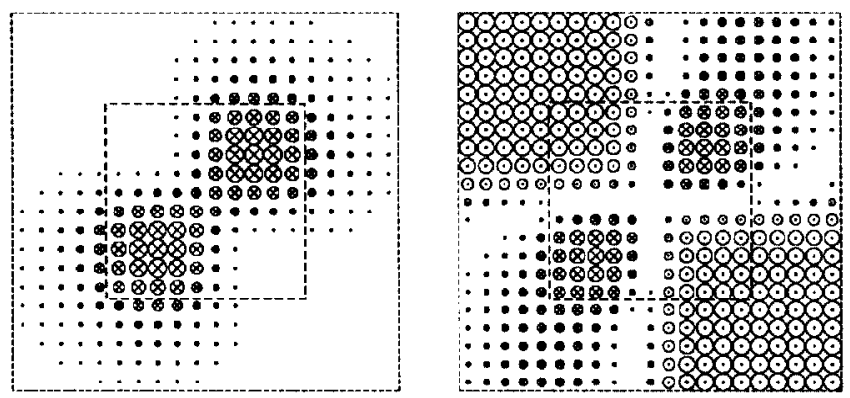

Fig. 7: MAFIA plot of $\vec{E}$ field in the gap (detail)

$E_{z}$ component in the gap. In principle, there are no limitations to reach an ideal tuning.

The solution shown in Fig. 6 (two strips and two plates) gave quite good results: Fig. 7 shows $\vec{E}$ field in a transverse section near end plate for the initial configuration (left) and for the modified geometry (right); notice $E_{z}=0$ on the axis. The smaller squares define the "interesting" region for beam dynamics.

\section{MEASUREMENTS}

To check proof of principle we used the geometry of Fig. 6, but it should be noted that in practice it is necessary to change the shape of the last stems, in order to increase $L_{\text {add }}$ in fact, as the end plate is very close to the electrodes, it is not easy to reach an high value of $L_{\text {add }}$ with a reasonable shape. Besides, large plates should be avoided.

The electric field between end plate and each electrode was measured on a cold model, using a perturbing bead; Fig. 8 shows normalized $\left|E_{z}\right|$ field measured on the model in its initial configuration (left) and after the tuning (right); here \#1 and \#3 are the "hot" electrodes. These results confirm the situation described by MAFIA.

Bead-pull measurements of the inter-electrode voltage all along the structure were performed on the cold model, using an automatic computer-controlled system [6]. The results before and after tuning are shown in Fig. 9; a comparison with MAFIA simulations (continuous lines) emphasizes a quite good agreement, the small discrepancy being due to the slightly different position of the bead during measurements with respect to the path used in simulation, and to mechanical misalignments of the electrodes.
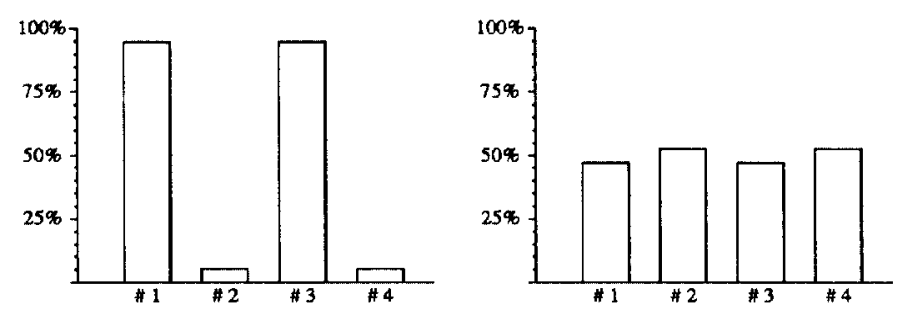

Fig. 8: Normalized $|\vec{E}|$ field in the gap
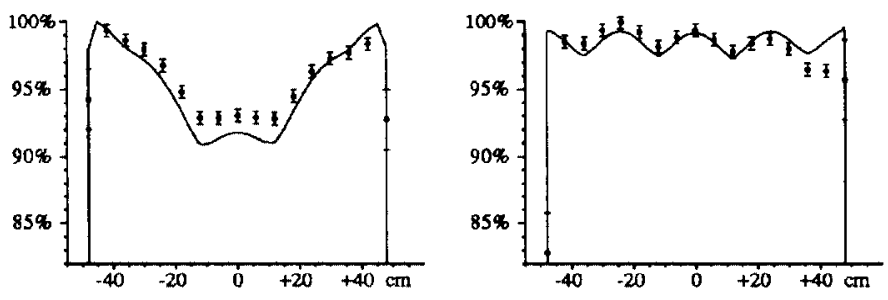

Fig. 9: Inter-electrode voltage distribution $V(z) / V_{e}$

\section{CONCLUSIONS}

The main result of this study is that both a good voltage symmetry in the region between end plate and electrodes and a good longitudinal inter-electrode voltage distribution can be reached by proper tuning of the end cells.

A more detailed analysis of the phenomenon is still needed to find a shape that could be used in practice, under working conditions (high power and vacuum problems): the aim of this paper is just to give hints of possible solutions and proof of principle of the adopted methodology.

\section{ACKNOWLEDGMENTS}

The authors are very grateful to W. Pirkl and M. Weiss for their essential help in understanding the phenomenon, and to A.M. Porcellato for her assistance.

\section{REFERENCES}

[1] A. Lombardi, G. Parisi, M. Vretenar, "Comparison Study of RFQ Structures for the Lead-Ion Linac at CERN", Proc. 1992 European Particle Acc. Conf., Berlin, Germany, March 1992, pp. 557-59.

[2] R. Klatt et al., "MAFIA - A Three-dimensional Electromagnetic CAD System for Magnets, RF Structures and Transient Wake-field Calculations", Proc. 1986 Linear Acc. Conf., Stanford, California, June 1986, pp. 276-78.

[3] A. Schempp, H. Deitinghoff, M. Ferch, P. Junior, H. Klein, "Four-Rod- $\lambda / 2-R F Q$ for Light Ion Acceleration", Nucl. Instr. and Meth. in Phys. Res., B10/11, 1985, pp. 831-34.

[4] M. Comunian, A. Pisent, "Longitudinal Dynamics at the Input of a Four-Rods RFQ", LNL-INFN Internal Note (REP) 61/92, April 1992.

[5] W. Pirkl, "Balancing the End Cell of a 4-Rod RFQ", CERN-PS Informal Note, March 1992.

[6] A.Porcellato, A.Battistella, S.Marigo, "Automation of the Cavity Parameter Measurements", Proc. 1990 European Particle Acc. Conf., Nice, France, June 1990, pp. 1109-11. 